University of Nebraska - Lincoln

DigitalCommons@University of Nebraska - Lincoln

$5-2009$

\title{
Modeling parasitism rate and parasitism risk: An illustration using a colonially nesting songbird, the red-winged blackbird Agelaius phoeniceus
}

\author{
Max Post van der Burg \\ University of Nebraska-Lincoln,, maxpostvanderburg@usgs.gov \\ Larkin A. Powell \\ University of Nebraska-Lincoln, Ipowell3@unl.edu \\ Andrew J. Tyre \\ University of Nebraska-Lincoln, atyre2@unl.edu
}

Follow this and additional works at: https://digitalcommons.unl.edu/natrespapers

Part of the Behavior and Ethology Commons, Ornithology Commons, and the Poultry or Avian Science Commons

Post van der Burg, Max; Powell, Larkin A.; and Tyre, Andrew J., "Modeling parasitism rate and parasitism risk: An illustration using a colonially nesting songbird, the red-winged blackbird Agelaius phoeniceus" (2009). Papers in Natural Resources. 364.

https://digitalcommons.unl.edu/natrespapers/364

This Article is brought to you for free and open access by the Natural Resources, School of at DigitalCommons@University of Nebraska - Lincoln. It has been accepted for inclusion in Papers in Natural Resources by an authorized administrator of DigitalCommons@University of Nebraska - Lincoln. 


\title{
Modeling parasitism rate and parasitism risk: An illustration using a colonially nesting songbird, the red-winged blackbird Agelaius phoeniceus
}

\author{
Max Post van der Burg, Larkin A. Powell and Andrew J. Tyre \\ School of Natural Resources, University of Nebraska-Lincoln, 228 Hardin Hall, Lincoln, Nebraska, 68583-0982, USA \\ Corresponding author - Max Post van der Burg; present address: Department of Ecology, Evolution and Organismal Biology, \\ lowa State University, 228 Bessey Hall, Ames, lowa, 50011-1020, USA; email: mpost@iastate.edu
}

\begin{abstract}
Ornithologists interested in the drivers of nest success and brood parasitism benefit from the development of new analytical approaches. One example is the development of so-called "log exposure" models for analyzing nest success. However, analyses of brood parasitism data have not kept pace with developments in nest success analyses. The standard approach uses logistic regression which does not account for multiple parasitism events, nor does it prevent bias from using observed proportions of parasitized nests. Likewise, logistic regression analyses do not capture fine scale temporal variation in parasitism. At first glance, it might be tempting to apply log exposure models to parasitism data, but the process of parasitism is inherently different from the process of nest predation. We modeled daily parasitism rate as a Poisson process, which allowed us to correct potential biases in parasitism rate. We were also able to use our estimated parasitism rate to model parasitism risk as the probability of one or more parasitism events. We applied this model to red-winged blackbird Agelaius phoeniceus nesting colonies subject to parasitism by brown-headed cowbirds Molothrus ater. Our approach allowed us to model parasitism using a wider rage of covariates, especially functions of time. We found strong support for models combining temporal fluctuations in parasitism rate and nest-site characteristics. Similarly, we found that our annual predicted parasitism risk was lower on average than the risk estimated from observed parasitism levels. Our approach improves upon traditional logistic regression analyses and opens the door for more mechanistic modeling of the process of parasitism.
\end{abstract}

Ornithologists have been fascinated by the mechanisms driving variation in nest success and brood parasitism for many decades (Mayfield 1961, Lack 1968). Some biologists treat survival and parasitism as binomial responses and thus use logistic regression to evaluate the influence of covariates on these phenomena (e.g. Zanette and Jenkins 2000, Davidson and Knight 2001, Antonov et al. 2007). These analyses categorize nests as either successful or failed, and parasitism is either present or absent in a nest. Recently, researchers have recognized that using logistic regression to analyze nest success is limited because it relies on apparent success (i.e. observed proportions of successful nests), which may result in biased estimates (Dinsmore et al. 2002, Hazler 2004, Shaffer 2004). Mayfield $(1961,1975)$ recognized that apparent estimators of nest success tended to overestimate nest success because older nests were more likely to survive and nests that survived for a short time were less likely to be found. As a solution, Mayfield suggested the use of exposure days to estimate a daily survival rate that could be used to estimate an accurate probability of nest success. Building on the notion of exposure days, researchers adjusted logistic regression models, so-called "log exposure" models, which produce estimates similar to Mayfield's approach (Rotella et al. 2004). Improved logistic regression models have allowed ornithologists to analyze nest success with an increasing array of model covariates, including fine scale temporal effects (e.g. Grant et al. 2005).

Advances in nest survival analysis have not been applied to the analysis of parasitism data. Many parasitism analyses assess the effects of covariates using the entire nest cycle as the response period, instead of observation intervals. Thus, time-specific covariates on the order of days have not been available to biologists. Some analyses also ignore multiple parasitism events when estimating parasitism rates (e.g. Budnik et al. 2002, Sharp and Kus 2006). However, Banks and Martin (2001) accounted for multiple parasitism events by treating parasitism rate as the number of eggs laid per day.

A proper definition of "parasitism rate" is critical for proper analysis of and drawing inference from parasitism data. Many biologists have used the term "rate" to describe the proportion of nests parasitized (e.g. Brown and Lawes 2007, Dyrcz and Halupka 2007, Hoover and Robinson 2007), but we believe that rate should have a temporal unit of measurement. To that end, Powell and Knutson (2006) suggest that parasitism proportion would be best described as "parasitism level." Other biologists refer to the percentage of ob- 
served nests as "probability of parasitism" and "frequency of parasitism" (McLaren and Sealy 2003, Jensen and Cully 2005). Here, like Banks and Martin (2001), we define parasitism rate as the number of parasitic eggs that a nest receives per day. A clear definition of parasitism rate also clearly defines "parasitism risk." We define parasitism risk as the probability of a nest being parasitized at least once during the incubation stage. We present a method of estimating parasitism risk from the probability of one or more parasitism events.

The simplest approach to assigning parasitism status to a nest is to treat the nest with a binomial response (parasitized, not parasitized). However, many studies report multiple parasitism events, and biologists often treat this separately as parasitism intensity (e.g. McLaren and Sealy 2003, Jensen and Cully 2005, McLaren et al. 2006). Logistic regression, based on the binomial response, fails to incorporate the additional information about the rate contained in multiple parasitism events. Additionally, normal nest searching is prone to miss parasitism events that are masked by early nest mortalities, causing bias in the observed proportion of parasitized nests.

The study of factors associated with parasitism rates needs a more flexible modeling approach that allows for increased temporal detail, accounts for multiple parasitism events and takes into consideration the issue of bias in using observed proportions. At first glance, it might be tempting to treat parasitism events like predation events and apply log exposure nest survival models (Rotella et al. 2004) to parasitism data. But parasitism data are unique. Once a nest mortality event occurs, a nest cannot reenter an analysis and the data are right censored. In contrast, nests continue to be active following parasitism events and other parasitism events may occur. Thus, the nest should not be right-censored after a parasitism event.

One way to deal with this problem is to redefine what parasitism rate means in light of how parasitism events occur. Parasites lay eggs as discrete events which occur with a measurable daily frequency. For species like cowbirds, multiple eggs are often laid within a single observation interval. Cuckoos Cuculus canorus, on the other hand, appear to vary more in terms of how frequently they multiply parasitize their hosts (Moskat and Honza 2002, Honza and Moskat 2005). In studies involving nest monitoring, this frequency can be measured as the number of new eggs laid during each monitoring interval. Thus, we suggest that parasitism rate, as we have defined it, would be better represented as a Poisson process rather than a binomial process. A Poisson process is a count of events that occur with a fixed rate within a specified time interval. The resulting mean of a Poisson distribution is the average rate at which the event occurs. Whether this particular distribution would continue to work for analyses of data in which multiple parasitism events are rare or absent would have to be evaluated. Regardless, the real advantage of our approach is that, like nest survival analyses, one can account for bias in observed parasitism events by modeling parasitism rate as a daily process using interval data. Likewise, this daily rate can be used to estimate on an overall risk that is less biased than using the observed proportion of nests.
Our goal in this paper is to develop a modeling approach that accounts for the number of days a nest is exposed to possible parasitism events, while treating the rate of parasitism as a Poisson process. Our objectives are to: (1) estimate parasitism rate, and (2) use the parasitism rate to estimate parasitism risk. We demonstrate the use of this model by analyzing data from red-winged blackbird Agelaius phoeniceus nests suffering parasitism by brown-headed cowbirds Molothrus ater.

\section{Methods}

\section{Target species}

The red-winged blackbird (hereafter, blackbird) is a habitat generalist that nests in loose colonies and is considered one of the more common hosts available to the brownheaded cowbird (Ortega and Cruz 1988, Yasukawa and Searcy 1995). Blackbirds are considered parasite egg "acceptors" (Ortega and Cruz 1988). The apparent acceptance behavior of red-winged blackbirds has been interpreted as a strategy that lowers the chance of nest failure following parasitism as rejection may be costly for some hosts because the act of trying to remove the parasite eggs could damage host eggs (Ortega and Cruz 1988, Beletsky 1996). Hosts that accept parasite eggs in order to reduce this risk may then experience a reduction in reproductive output (e.g. smaller host clutch size or lower host fecundity), rather than outright failure of the nest (Ortega and Cruz 1988; Beletsky 1996).

Cowbirds are generalist brood parasites that parasitize a wide variety of different hosts (Lowther 1993, Johnsgard 1997). Although blackbirds are not always regarded as preferred hosts for cowbirds (Woolfenden et al. 2004), they do experience moderate amounts of parasitism, which likely causes some loss of host fitness through egg removal by cowbirds (Freeman 1990, Rothstein 1990).

Because of this potential loss of fitness, blackbirds should choose nesting strategies that minimize both the risk of parasitism, while also trying to avoid the risk of nest predation (Rothstein 1990). For example, research suggests that blackbirds can reduce their risk of parasitism by nesting in larger colonies or by aggregating nests (Clotfelter 1998, Clotfelter and Yasukawa 1999a, Strausberger 2001), placing nests in locations that reduce parasite searching efficiency (Freeman 1990, Clotfelter 1998), or by timing nesting within the season when alternate hosts are more available (Woolfenden et al. 2004).

\section{Study area}

We conducted our study in the Rainwater Basin region of central Nebraska. The Rainwater Basin is a complex of playa wetlands that occupies roughly $10,800 \mathrm{~km}^{2}$ in south-central Nebraska. We studied blackbird nesting colonies within 10 different wetlands in a 13- by $18-\mathrm{km}$ area southeast of Clay Center, Nebraska in Clay County. Dominant plant communities within wetlands were composed of cattail Typha spp., river bulrush Scirpus fluvailtalis, reed canary grass Phalaris arundinacea and smartweed Polygonum spp. 
Nest monitoring

We searched for nests from mid-May through early August during the 2002-2004 breeding seasons. We searched entire wetlands with area $<1$ ha. On wetlands with area $>1$ ha, we searched for nests within $100 \mathrm{~m}$ of a randomly established transect. We marked nests with plastic flagging tied to vegetation $>10 \mathrm{~m}$ from the nest. We visited nests every 3-4 d until the young successfully fledged or the nest failed. At each visit we recorded the number of eggs or nestlings (both host and parasite). If the clutch was finished before we found the nest we were not able to determine the number of new parasite eggs laid since we could not assign them to an observation interval. Using information from hatching and laying events we were often able to approximate when a nest was initiated. We also recorded Universal Transverse Mercator (UTM) coordinates for each nest using a WAAS-ready Garmin Etrex Vista global positioning system (GPS) receiver.

To assess the effect that colonial nesting may have on parasitism rates, we calculated the number of simultaneously active blackbird nests as the number of nests, regardless of stage, during each nest observation interval within each colony. We also calculated the shortest distance between simultaneously active blackbird nests, as an index of nest aggregation. To determine the role of nest-site characteristics, we measured both the shortest distance between each nest and the edge of the wetland with ArcGIS 8.3 (ESRI) and nest height. To assess the role of temporal fluctuations in parasitism risk we calculated an average ordinal day in the breeding season and a nest-specific age for the mid-point of each observation interval. We included the entire laying and incubation periods, as we observed parasitism events as late as 15 days past the date when the first egg was laid.

\section{Statistical analyses}

Our analyses were performed using the statistical computing language R 2.6.0 (R Development Core Team 2007). To estimate the daily parasitism rate for our sample of nests we used generalized additive models (GAM) with a log-link function which we used to estimate $\lambda$, the Poisson mean (rate of parasitism events per $\mathrm{d}$ ). In the model fitting we used the offset term $\log (t)$, to account for the length of the observation interval and thus ensure that $\lambda$ was estimated as a daily rate. Our estimated daily rate can be extrapolated to estimate the rate over a particular observation interval of length $t$. The number of new parasite eggs laid per observation interval was the response variable, allowing us to predict daily parasitism rates $(\lambda)$ as well as overall parasitism risk $(\varphi)$.

We developed 4 base models: (1) colonial effects, (2) nest site, (3) temporal, and (4) null model. Our colonial effects model included parameters that described the number of simultaneously active blackbird nests (Sim) and the distance to the closest simultaneously active blackbird nest (Dist). Our nest-site model included effects of the distance of each nest from the wetland's edge (Edge) and the height of each nest $(\mathrm{Ht})$. Our temporal model included effects of the average or- dinal day in the breeding season (Day), the average age of each nest based on our approximated initiation days (Age) and year (Y). Finally, our null model (Constant) had only an intercept. To keep the total number of models small, but still investigate combinations of effects, we built pair-wise combinations of the above models and a global model with all the parameters. We also included six additional models each composed only of a main effect.

In total, we fit 15 models using a penalized spline regression algorithm, allowing us to investigate non-linear effects of the parameters in each model (Wood and Augustin 2002). All the parameters for continuous variables were considered as smooth terms in the models except year which was a categorical variable. We used the R package mgcv to fit each model. This package uses iterative least squares to fit each model and treats the number of smoothing terms as something to be estimated in the context of model fitting. The spline terms for each parameter are chosen based on how well they balance model fit and overall smoothness (Wood and Augustin 2002). The result is a model that has more "wiggliness" than a GLM, but is not overfitted. If a model parameter fits better as a spline term than the degrees of freedom will increase. The number of parameters in each model is approximated by the effective degrees of freedom (edf); fixed effects (non-spline terms) contribute only one degree of freedom.

We used information theoretic methods to rank candidate models and to account for model uncertainty. Because information theoretic methods make specific assumptions about prior beliefs in model complexity the choice of criterion should explicitly reflect a researcher's view of reality (Link and Barker 2006). We chose to use Bayes information criterion (BIC), because it favors simpler models (Link and Barker 2006). We compared our chosen model selection criterion against the commonly used AIC (Akiake's information criterion), which we expected to favor more complicated models.

We should note that our choice of information criterion is not arbitrary, but rather reflects a choice of whether prior belief is implicit or explicit in our model selection exercise. Where this may become an issue is for AIC; the socalled "Kullback-Leibler prior" on AIC weights essentially changes as sample size increases (Link and Barker 2006), thus the data may not overwhelm the prior belief. Additionally, because of AIC's sensitivity to sample size, the increase in model parameters has a tendency to increase the expected value of the model probability. Using weighted BIC, as we have here, allowed us to explicitly state our prior belief and thus allow the data to influence our posterior inference.

We specified our prior belief in our models as a uniform distribution (i.e. $\operatorname{pr}\left(\right.$ model $\left.\left._{\mathrm{i}}\right)=1 / 15\right)$, which means that we did not favor any one model or set of models. We considered our best model to be the one with the highest posterior model probability (Link and Barker 2006). Note that we use the term "model probability" in the same sense as the more commonly used term "model weight" (Burnham and Anderson 2002, Link and Barker 2006). We then checked the adequacy of our best model(s) using k-fold cross validation. This technique of model checking requires that a data set be randomly broken into $k$ subsets. Then, $k-1$ of the sub- 
sets are used to "train" the model by estimating the parameters of the best model, which is then used to predict the data in the subset that was withheld. In our case we used 8 folds or subsets because it allowed us to break our data up evenly. The result was that we used each subset once as testing data and each data point appeared in the training data seven times. We assessed how well the subset was predicted by using a calibration curve. This involved taking the linear predictors from the withheld subset and using them as covariates (on the linear scale) in a linear Poisson model in which the response was the number of new parasite eggs observed in the withheld subset. If the model adequately predicted the withheld data than the linear Poisson model should have an intercept of 0 and a slope of 1 . We calculated the mean and 95\% CI (SE × 1.96) of the intercept and slope from the eight testing runs. We considered a model a good fit if the confidence intervals overlapped 0 and 1 for our intercept and slope terms respectively. Following model selection and checking we made predictions for each continuous model parameter by holding all the other parameters at their mean values and allowed the parameter of interest to vary over the range observed in the field.

Since parasitism rate depends on the number of exposure days, parasitism risk for a nest can be estimated as:

$$
\hat{\varphi}_{j}=1-\prod_{i=1}^{\mathrm{I}}\left(\mathrm{e}^{-\lambda t_{i j}}\right)
$$

where $i$ is the interval, $j$ is the nest and $t$ is the number of exposure days.

To compare our method of estimating parasitism risk to observed parasitism levels we used a non-parametric bootstrapping method with our data. The goal in this analysis was to demonstrate how our modeling approach can be used to correct the bias in our risk estimate compared with uncorrected observed parasitism levels. We sampled nests from our dataset according to the number of nests we observed over all three years. We used our best model to estimate parasitism risk for each sample of nests. We performed this analysis 1,000 times and then estimated the mean risk by calculating the mean of the distribution of parasitism risk estimates and the $95 \%$ confidence intervals by calculating the 0.025 and 0.975 quantiles of the distribution. We estimated overall parasitism risk (probability of parasitism during life of the nest) assuming a nest was exposed for $15 \mathrm{~d}$. We assumed this amount of time because the maximum average age we estimated (i.e. the parameter Age) was $15 \mathrm{~d}$ old.

For each sample of nests we also estimated the parasitism level (P): If the sampled nest was parasitized at least once we assigned the nest a one; if the nest was not parasitized at all we assigned the nest a zero $\left(\mathrm{P}_{j}=\{0,1\}\right)$.

We then estimated parasitism level as:

$$
\hat{\mathrm{P}}=\frac{1}{\mathrm{~K}} \sum_{k=1}^{\mathrm{K}}\left(\frac{1}{\mathrm{~J}} \sum_{j=1}^{\mathrm{J}} \mathrm{P}_{j k}\right)
$$

where $j$ is the nest and $k$ is the iteration.

\section{Results}

We monitored 592 blackbird nests from 2002-2004. The parasitism level over all three years was 31\% (SE 1.9 percentage points, $n=592$ ); parasitism level also varied during the course of our study (2002: $48 \%$; SE 4.6 percentage points, $n=$ 117, 2003: $23 \%$; SE 3.2 percentage points, $n=166$, 2004: $28 \%$; SE 2.6 percentage points, $n=309$ ). In addition, there was considerable variation between wetlands over the three years; parasitism levels were as low as $0 \%$ and as high as $58 \%$. Parasitized nests contained an average of $1.71(\mathrm{SD}=1.15$, range =1-7) parasite eggs and had a lower average host clutch size $($ mean $=2.07, \mathrm{SD}=1.31)$ compared to unparasitized nests (mean $=3.16, \mathrm{SD}=1.07, \mathrm{t}=-9.69, \mathrm{P}<0.001)$. Blackbird colonies, on average, contained about $14.50(\mathrm{SD}=11.20)$ nests over the three years. We also observed that $43 \%$ of new parasitism events occurred between 2-4 d after nest initiation; only $14 \%$ occurred $10-15 \mathrm{~d}$ after initiation.

For our analysis, we restricted our sample to 368 nests because we were not able to accurately estimate the average ages of all our nests. We considered our reduced sample representative for this species and region because the estimated parasitism level was similar to the full sample $(26 \% ; \pm 2.3 \mathrm{SE}, n=368)$. Using BIC as our selection criterion, the model with the highest probability contained both temporal and nest-site effects and there was weak support for models with colonial effects (Table 1). Our model checking efforts suggested that this model fit the data well (intercept: mean $=-0.29,95 \%$ CI: $-2.55-1.97$; slope: mean $=0.94,95 \%$ CI: 0.28-1.61). Using AIC, we found that the most complex model had the highest model probability (Table 1). Therefore, under the use of AIC there was more support for colonial effects. This model also seemed to fit the data fairly well (intercept: mean $=0.85,95 \%$ CI: $-2.74-1.90$; slope: mean $=0.79,95 \%$ CI: $0.24-1.34)$. We made model predictions under the model chosen by BIC for two reasons. First, the parameters estimated in the model chosen under BIC had the same qualitative effects as those same parameters that appeared in the model under AIC. However, the predicted effect of all the model parameters was fairly weak under the AIC model. Second, the BIC model was simpler and was chosen under the assumption that there was no prior preference in this model.

We present parameter estimates for linear effects from the best model in Table 2 . Predicted daily parasitism rates $(\lambda)$ varied among years with the rate being higher in $2002(\lambda=$ $0.021,95 \%$ CI: $0.011-0.041)$ compared with $2003(\lambda=0.007$, 95\% CI: $0.003-0.016)$, and $2004(\lambda=0.013,95 \%$ CI: $0.007-$ 0.023 ). Again, these predictions were made holding the continuous variables in the model at their mean values. In an exploratory analysis we fit an additional model with interaction terms for year by nest site and year by time effects, which had a posterior model probability of zero. Model predictions from the best model also show that nest site and time effects were qualitatively similar between years. Thus, for the sake of space, we present our predictions with the year parameter set to 2002. Daily parasitism rates varied non-linearly as nests aged, with the highest rate at an average age of about $3 \mathrm{~d}$ (Figure 1A). Ordinal day of the nesting season showed a negative effect on daily parasitism rate 
Table 1. A comparison of candidate models explaining daily rate of parasitism $(\lambda)$ for 368 red-winged blackbird nests in the Rainwater Basin region of Nebraska for 2002-2004. These models compared effects of age, ordinal day of the breeding season (d), year (y), nest height (ht), distance from wetland edge (edge), number of (sim), and distance between (dist) simultaneously active blackbird nests within a colony. The number of parameters $(k)$ includes an intercept plus the approximate degrees of freedom for the spline function. The posterior probability for each model is approximated using the BIC score of each model, a uniform prior and Bayes' theorem. For comparison we also included AIC scores and weights $\left(\mathrm{AIC}_{\mathrm{w}}\right)$ for each model.

\begin{tabular}{lrrcrc}
\hline Model & $\mathrm{k}$ & $\mathrm{BIC}$ & $\begin{array}{c}\text { Model } \\
\text { probability }\end{array}$ & $\mathrm{AIC}$ & $\mathrm{AIC}_{\mathrm{w}}$ \\
\hline$\lambda_{\text {age+d+y+ht+edge }}$ & 11.3 & 523.9 & 0.99 & 479.7 & 0.00 \\
$\lambda_{\text {age+d+y+sim+dist }}$ & 19.0 & 533.8 & 0.01 & 459.7 & 0.04 \\
$\lambda_{\text {global }}$ & 20.9 & 535.2 & 0.00 & 453.3 & 0.96 \\
$\lambda_{\text {age+d+y }}$ & 9.5 & 540.3 & 0.00 & 503.0 & 0.00 \\
$\lambda_{\text {ht+edge }}$ & 3.0 & 546.6 & 0.00 & 534.9 & 0.00 \\
$\lambda_{\mathrm{d}}$ & 2.7 & 548.3 & 0.00 & 539.4 & 0.00 \\
$\lambda_{\mathrm{y}}$ & 3.0 & 549.7 & 0.00 & 537.9 & 0.00 \\
$\lambda_{\text {age }}$ & 6.7 & 550.4 & 0.00 & 524.2 & 0.00 \\
$\lambda_{\text {edge }}$ & 2.0 & 551.3 & 0.00 & 543.5 & 0.00 \\
$\lambda_{\text {sim+dist+ht+edge }}$ & 10.7 & 557.2 & 0.00 & 515.3 & 0.00 \\
$\lambda_{\text {ht }}$ & 2.0 & 563.4 & 0.00 & 555.6 & 0.00 \\
$\lambda_{\text {sim }}$ & 8.0 & 565.3 & 0.00 & 533.9 & 0.00 \\
$\lambda_{\text {sim+dist }}$ & 8.9 & 566.0 & 0.00 & 530.9 & 0.00 \\
$\lambda_{\text {constant }}$ & 1.0 & 567.0 & 0.00 & 563.1 & 0.00 \\
$\lambda_{\text {dist }}$ & 2.0 & 569.1 & 0.00 & 561.3 & 0.00 \\
\hline
\end{tabular}

(Figure 1B) as did distance from wetland edge (Figure 1C). Nest height showed a similar negative effect, but was not as strong as the other effects (Figure 1D).

Our estimate for overall parasitism risk from our bootstrapping analysis was 16\% (95\% CI: 15\%-18\%); the estimate of parasitism level was $26 \%$ (95\% CI: $21 \%-30 \%)$. Using our best model we also estimated annual variation in overall parasitism risk while holding the other model parameters at their means (2002: 27\%; 95\% CI: 15\%-46\%, 2003: 10\%; 95\% CI: 5\%-21\%, 2004: 17\%; 95\% CI: 10\%-29\%). Because our best model included effects of time on parasitism rate, we estimated parasitism risk conditional on day of nest initiation, which gave us the overall probability of a nest being parasitized before completion of incubation. Our results (Figure 2) showed that parasitism risk was high for nests initiated earlier in the breeding season, but that this risk declined for nests initiated later in the season. The magnitude of this decline depends on the height of each nest, as well as its location relative to wetland edge.

\section{Discussion}

Our observed parasitism levels were consistent with reported levels for prairie nesting red-winged blackbirds (30\%-41\%; Weatherhead 1989). However, if nests are found at various stages of development throughout the nesting season, there is potential for bias which may mask true variation caused by changes in cowbird behavior. A key advantage of the interval rate model we used is that it controls for variation in when nests are found, thus correcting for the potential bias in using observed proportions of nests. Furthermore, one would not expect parasitism rates to be tem-
Table 2. Parameter estimates from the best model explaining daily rate of parasitism for 368 red-winged blackbird nests in the Rainwater Basin region of Nebraska for 2002-2004. This model contained effects of year (y), nest age (age), ordinal day of the breeding season (d), distance from wetland edge (edge), and nest height (ht). We present estimates for model terms determined to have linear effects on parasitism rate as the mean values with standard errors (SE) in parentheses. Age was the only parameter with a spline term and is not presented.

\begin{tabular}{lc}
\hline Parameter & Estimate (SE) \\
\hline Intercept & $-1.33(0.46)$ \\
Y: 2003 & $-1.06(0.39)$ \\
Y: 2004 & $-0.52(0.26)$ \\
Age & - \\
Day & $-0.02(0.01)$ \\
Edge & $-0.02(0.005)$ \\
Ht & $-0.89(0.76)$ \\
\hline
\end{tabular}

porally constant and our approach allowed us to model variation in parasitism rate and risk as functions of daily processes as well as site-specific processes with little or no temporal variation.

Others have compared multiple sources of variation in the likelihood of parasitism and have reported effects of colonial nesting patterns (Massoni and Reboreda 2001, Brown and Lawes 2007). But these studies were based on observed parasitism levels and did not compare models with factors that varied by day and for longer periods of time. We found no support for colonial nesting dynamics on parasitism rate in our study system, considering the simplest model.

Our results suggest the presence of other sources of variation in parasitism rate, particularly with regard to effects of time. Parasitism rates varied between years; a finding consistent with other patterns of observed parasitism for this host species (Beletsky 1996, Clotfelter and Yasukawa 1999b). At a finer temporal scale, nest age shows a non-linear effect that suggests a peak rate of parasitism as the host nears completion of its clutch. A likely explanation could be that adult cowbirds prefer nests with more host eggs (White et al. 2007) and thus preferentially parasitize nests with complete clutches. This is an assertion that needs to be tested, but our findings do suggest the presence of a possible cue that cowbirds may use to decide whether to deposit eggs. White et al. (2007) found that juvenile cowbirds seemed to prefer nests that had already been parasitized, which could explain why we find parasitism events so late in the incubation period (i.e. inexperienced laying). However, this explanation requires that cowbirds are able to detect host nests fairly soon after clutch completion, or that they monitor several nests and parasitize whichever none is at the right stage of development. This timing is critical if a cowbird expects its eggs to be incubated the necessary length of time for successful hatching. Interestingly, blackbirds are not viewed as preferred hosts for cowbirds probably because their eggs both require similar amounts of time for incubation and fledging (Woolfenden et al. 2004). This could make cues for assessing the appropriate time for parasitizing blackbird nests extremely important.

Freeman et al. (1990) reported that cowbirds occasionally "dumped" eggs in inactive blackbird nests presumably because they were unable to assess how old a nest was. 

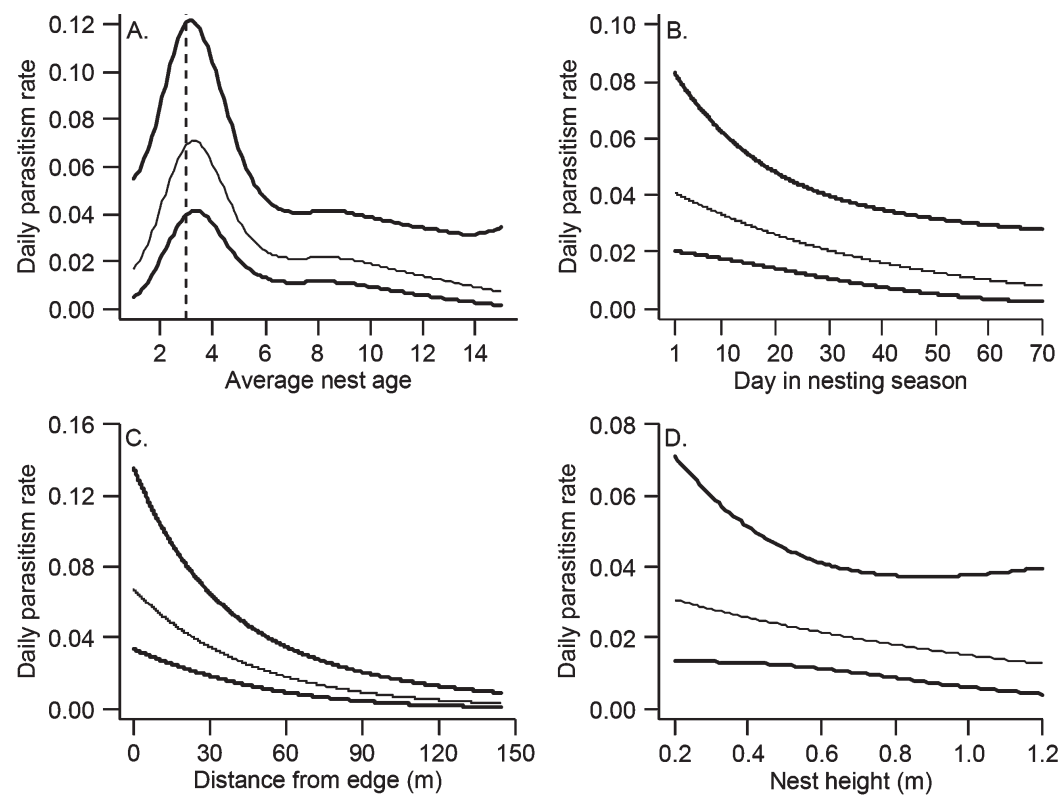

Figure 1. Predicted trends in the daily parasitism rate $(\lambda$, no. of parasite eggs per $d)$ for red-winged blackbirds nesting in the Rainwater Basin region of Nebraska, USA in 2002. We chose the trends we predicted based on the best model explaining variation in parasitism rates. These trends were estimated as functions of $(A)$ average age of the nest ( 1 1= first egg laying; $d 3=$ average onset of incubation), (B) ordinal day in the nesting season (d 1 = May 14), (C) distance from wetland-upland edge, and (D) nest height. We predicted each trend by allowing one parameter to vary while holding the other parameters at their mean values. Thin lines represent average rates and the thick lines represent $95 \%$ confidence intervals. The vertical dotted line in panel $\mathrm{A}$ is the average onset of incubation.
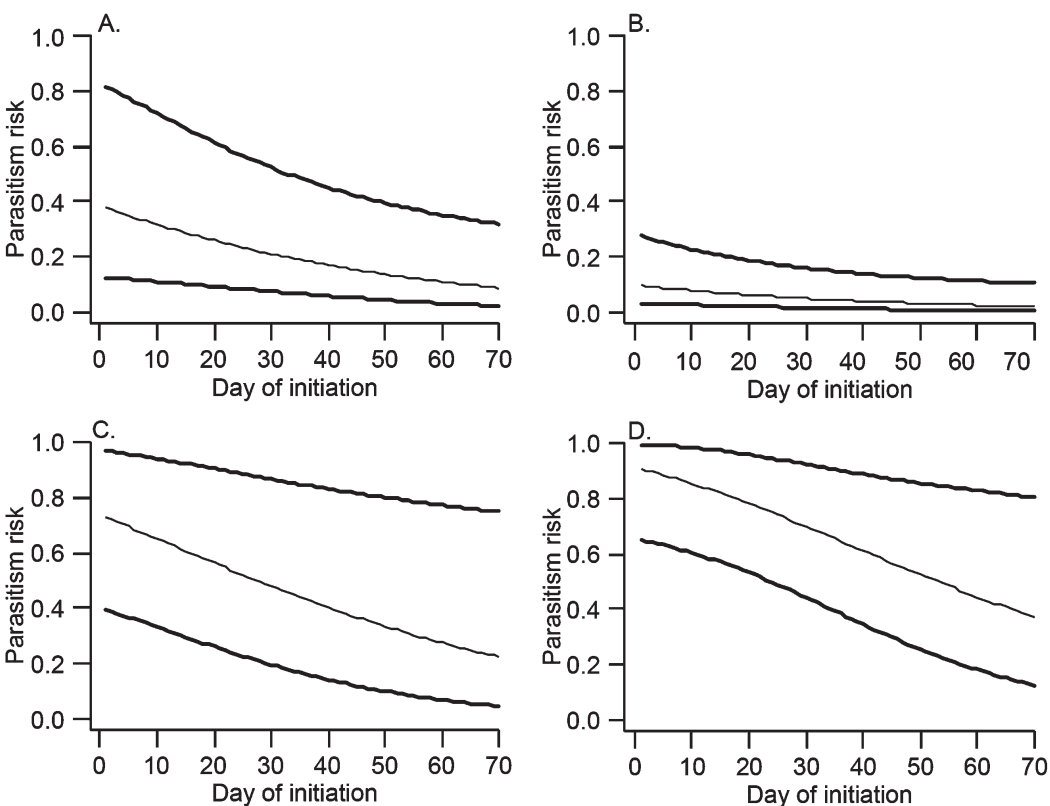

Figure 2. Predicted trends in parasitism risk ( $\varphi$, probability that a nest will be parasitized $\geq 1$ time) for red-winged blackbirds nesting in the Rainwater Basin region of Nebraska, USA in 2002. Parasitism risk was estimated as a function of the ordinal day the nest was initiated assuming $15 \mathrm{~d}$ of exposure ( $\mathrm{d} 1=$ May 14). We predicted these trends based on the best model that explained variation in parasitism rates. These trends were predicted as functions of nest height $(\mathrm{A}: \mathrm{ht}=1.15 \mathrm{~m}, \mathrm{C}: \mathrm{ht}=0 \mathrm{~m})$ and distance from wetland-upland edge $(\mathrm{B}$ : dist $=143.6 \mathrm{~m}$, D; dist $=0 \mathrm{~m}$ ). We made predictions at the upper and lower values of our observed field data. The thin lines represent average risk and the thick lines represent $95 \% \mathrm{Cl}$.

They suggested that cowbirds were more accurate in timing their laying with the host when they had access to perches or other observation points. This so-called "inappropriate laying" may have been a last ditch attempt at reproduction (Freeman et al. 1990), and could explain why we found a slow decline in parasitism rate after the initial peak.

We also observed a decline in parasitism rate over the course of the breeding season. Other parasitism studies using the blackbird-cowbird system have found seasonal increases as well as seasonal decreases in parasitism (Freeman 
et al. 1990, Clotfelter and Yasukawa 1999a). Our observed variability in seasonal parasitism levels could be explained by variation in the availability of alternative hosts, with seasonal decreases indicating cowbirds switching to more preferred hosts (Fleischer 1986, Woolfenden et al. 2004).

Aside from temporal influences we also found relationships between parasitism rate and the spatial location of a nest. Nest position, relative to wetland edge, appears to influence parasitism rates with nests having higher rates near the wetland edge. Based on observations of cowbirds in uplands we feel it is likely that nests on the edges of wetlands are easier for searching cowbirds to find, probably because there are more perch sites in uplands. Interestingly, nests that are presumed to be more difficult to find (i.e. nests built closer to the ground; Martin 1993) appear to have higher parasitism levels. Clotfelter (1998) found no effect of the height of blackbird nests on parasitism level. Martin's (1993) analysis of parasitism levels in blackbirds found that taller nests had higher risk. One potential explanation for our results could be that shorter nests are more exposed or are more easily located by searching cowbirds. Anecdotal evidence from other systems in Nebraska show that some cowbirds search for potential nests from the ground (D. Kim, personal communication). On the other hand, if nests built earlier in the season are a higher risk of parasitism, and vegetation is shorter during this period, we might expect that nests closer to the ground might be at higher risk. Likewise, if vegetation matures differently at the edge of wetland compared with its interior, one might also expect higher parasitism rates at the edges of wetlands.

Using these predicted trends we were also able to estimate seasonal parasitism risk as a function of time and nest placement. Our results show that if we had relied on the observed proportion of parasitized nests to assess parasitism risk we would have on average overestimated parasitism risk and neglected an important temporal pattern in the fluctuation of such risk. The positive bias in our observed annual parasitism risk is a factor of the high rate of parasitism during onset of incubation (Figure 1A) and the fact that we did not find all nests as they were initiated. Thus, normal nest searching is prone to miss nesting events that are masked by early mortalities, causing positive bias in observed parasitism risk. Parasitism risk is markedly higher for nests near the edges of wetlands, suggesting that they are more prone to parasitism particularly early in the season. Similarly, lower nests are at a slightly higher risk than taller nests, but this risk changes in magnitude over the season. These results suggest that nests initiated early in the season, close to the ground and near wetland edges are more likely to be parasitized.

While our results display similar trends to those from other analyses, our ability to measure actual parasitism rates and nest specific parasitism risk is unique. And, our model translated parasitism rate to parasitism risk, which can be compared with observed levels of parasitism. One potential limitation of our approach is the fact that the host species may influence the researcher's ability to track parasitism events. For our host species, we believed this to be a fairly small problem since they accept parasite eggs. However, for species that remove parasite eggs, the researcher will have a more difficult time tracking exactly how many eggs were laid in each interval. One way to overcome this may be to adopt more continuous monitoring (i.e. every day) or to use nest cameras. Regardless, our results suggest that there may be patterns lurking in other data sets that may be found using alternative analytical approaches. We feel that a particularly interesting area to apply our approach would be to the question of host preference and host switching. Because of the potential for bias discussed earlier, using observed parasitism levels to infer host preference may be misleading. It would also be extremely useful to explicitly define whether host preference is defined based on the rate of parasitism (i.e. preferred hosts have higher rates) or whether overall risk between hosts is a better metric. Lastly, because host switching is really a problem of host preference over time, we feel that our approach provides an explicitly defined temporal model to begin answering such questions.

Our modeling approach facilitated the comparison of multiple working hypotheses about variation in brood parasitism rates within a Bayesian model selection framework. We were able to control for sampling variation as well as model non-linear effects of time on parasitism rate and risk. As parasitism events are discrete and happen on a daily basis, our approach accounts for variation in parasitism driven by the behavior of both host and parasite. Our results provide a critical step toward developing a more mechanistic model of factors driving parasitism rate and parasitism risk. Building on advances in the nest predation literature, we have provided biologists with a rigorous analytic method to investigate the intriguing dynamics of nest parasitism.

Acknowledgments - Funding was provided by the Layman Trust in the Institute of Agriculture and Natural Resources, as well as the School of Natural Resources at the University of NebraskaLincoln. M. Post van der Burg was supported by a GAANN graduate training grant from the Initiative for Ecology and Evolutionary Analysis at the University of Nebraska-Lincoln. Logistical and financial support was also provided by the U.S. Fish and Wildlife Service and the Nebraska Game and Parks Commission. T. Kocer, Z. Cunnigham, M. Lelevier, and N. J. Gonzalez assisted with field work. M. Giovanni, D. Kim, and J. Quinn provided constructive comments on early drafts of this manuscript. This research was supported by Hatch Act funds through the University of Nebraska Agricultural Research Division, Lincoln, Nebraska.

\section{References}

Antonov, A., Stokke, B. G., Moksnes, A. and Røskaft, E. 2007. Factors influencing the risk of common cuckoo Cuculus canorus parasitism on marsh warblers Acrocephalus palustris. J. Avian. Biol. 38: 390-393.

Banks, A. J. and Martin, T. E. 2001. Host activity and the risk of nest parasitism by brown-headed cowbirds. Behav. Ecol. 12: 31-40.

Beletsky, L. D. 1996. The red-winged blackbird: The biology of a strongly polygynous songbird. Acad. Press, San Diego.

Brown, M. and Lawes, M. J. 2007. Colony size and nest density predict the likelihood of parasitism in the colonial southern red bishop Euplectes orix-diderick cuckoo Chrysococcyx caprius system. Ibis 149: 321-327.

Budnik, J. M., Thompson, F. R. and Ryan, M. R. 2002. Effect of habitat characteristics on the probability of parasitism and predation of Bell's vireo nests. J. Wildl. Manag. 66: 232- 239. 
Burnham, K. P. and Anderson, D. R. 2002. Model selection and multimodel inference: A practical information-theoretic approach. Springer-Verlag, New York.

Clotfelter, E. D. 1998. What cues do brown-headed cowbirds use to locate red-winged blackbird host nests? Anim. Behav. 55: 1181-1189.

Clotfelter, E. D. and Yasukawa, K. 1999a. The effect of aggregated nesting on red-winged blackbird nest success and brood parasitism by brown-headed cowbirds. Condor 101: 729- 736.

Clotfelter, E. D. and Yasukawa, K. 1999b. Impact of brood parasitism by brown-headed cowbirds on red-winged blackbird reproductive success. Condor 101: 5-114.

Davidson, A. S. and Knight, R. L. 2001. Avian nest success and community composition in a western riparian forest. J. Wildl. Manag. 65: 334-344.

Dinsmore, S. J., White, G. C. and Knopf, F. L. 2002. Advanced techniques for modeling avian nest survival. Ecology 83: 3476-3488.

Dyrcz, A. and Halupka, K. 2007. Why does the frequency of nest parasitism by the cuckoo differ considerably between two populations of warblers living in the same habitat? Ethology 113: 209-213.

Fleischer, R. C. 1986. Brood parasitism by brown-headed cowbirds in a simple host community in eastern Kansas. Kans. Ornithol. Soc. Bull. 37: 21-29.

Freeman, S., Gori, D. F. and Rohwer, S. 1990. Red-winged blackbirds and brown-headed cowbirds: Some aspects of a hostparasite relationship. Condor 92: 336-340.

Grant, T. A., Shaffer, T. L., Madden, E. M. and Pietz, P. J. 2005. Time-specific variation in passerine nest survival: New insights into old questions. Auk 122: 661-672.

Hazler, K. R. 2004. Mayfield logistic regression: A practical approach for analysis of nest survival. Auk 121: 707-716.

Honza, M. and Moskat, C. 2005. Antiparasite behaviour in response to experimental brood parasitism in the great reed warbler: A comparison of single and multiple parasitism. Ann. Zool. Fenn. 42: 627-633.

Hoover, J. P. and Robinson, S. K. 2007. Retaliatory mafia behavior by a parasitic cowbird favors host acceptance of parasitic eggs. Proc. Natl. Acad. Sci. USA 104: 4479-4483.

Jensen, W. E. and Cully, J. F. 2005. Geographic variation in brownheaded cowbird (Molothrus ater) parasitism on dickcissels (Spiza americana) in great plains tallgrass prairie. Auk 122: 648-660.

Johnsgard, P. A. 1997. The avian brood parasites: Deception at the nest. Oxford Univ. Press, New York.

Lack, D. L. 1968. Ecological adaptations for breeding in birds. Methuen, London.

Link, W. A. and Barker, R. J. 2006. Model weights and the foundations of multimodel inference. Ecology 87: 2626- 2635.

Lowther, P. E. 1993. Brown-headed cowbird (Molothrus ater). In: Poole, A. and Gill, F. (eds.), Birds of North America, no. 47. Acad. Natl. Sci., Philadelphia and Am. Ornithol. Union, Washington.

Martin, T. E. 1993. Nest Predation among vegetation layers and habitat types: Revising the dogmas. Am. Nat 141: 897-913.

Massoni, V. and Reboreda, J. C. 2001. Number of close spatial and temporal neighbors decreases the probability of nest failure and shiny cowbird parasitism in colonial yellow-winged blackbirds. Condor 103: 521-529.
Mayfield, H. 1961. Nesting success calculated from exposure. Wilson. Bull. 73: 255-261.

Mayfield, H. 1975. Suggestions for calculating nest success. Wilson. Bull. 87: 456-466.

McLaren, C. M. and Sealy, S. G. 2003. Factors influencing susceptibility of host nests to brood parasitism. Ethol. Ecol. Evol. 15: 343-353.

McLaren, C. M., Underwood, T. J. and Sealy, S. G. 2006. Conflicting temporal changes in the frequency and intensity of cowbird parasitism on four common hosts in Ontario over 130 years. Condor 108: 238-242.

Moskat, C. and Honza, M. 2002. European cuckoo Cuculus canorus parasitism and host's rejection behaviour in a heavily parasitized great reed warbler Acrocephalus arundinaceus population. Ibis 144: 614-622.

Ortega, C. P., and Cruz, A. 1988. Mechanisms of egg acceptance by marsh-dwelling blackbirds. Condor 90: 349-358.

Powell, L. A. and Knutson, M. G. 2006. A productivity model for parasitized, multibrooded songbirds. Condor 108: 292-300.

R Development Core Team. 2007. R: A language and environment for statistical computing. R Found. Stat. Comput., Vienna.

Rotella, J. J., Dinsmore, S. J. and Shaffer, T. L. 2004. Modeling nestsurvival data: A comparison of recently developed methods that can be implemented in MARK and SAS. Anim. Biodivers. Conserv. 27: 187-205.

Rothstein, S. I. 1990. A model system for coevolution-Avian brood parasitism. Annu. Rev. Eco. Syst. 21: 481-508. Shaffer, T. L. 2004. A unified approach to analyzing nest success. Auk 121: 526-540.

Sharp, B. L. and Kus, B. E. 2006. Factors influencing the incidence of cowbird parasitism of least Bell's vireos. J. Wildl. Manag. 70: 682-690.

Strausberger, B. M. 2001. The relationship of habitat and spatial distribution of nests with brown-headed cowbird parasitism of red-winged blackbirds. Wilson. Bull. 113: 129-133.

Weatherhead, P. J. 1989. Sex ratios, host-specific reproductive success, and impact of brown-headed cowbirds. Auk 106: 358-366.

White, D., Ho, L., de los Santos, G. and Godoy, I. 2007. An experimental test of preferences for nest contents in an obligate brood parasite, Molothrus ater. Behav. Ecol. 18: 922-928.

Wood, S. N. and Augustin, N. H. 2002. GAMs with integrated model selection using penalized regression splines and applications to environmental modelling. Ecol. Model. 157: 157-177.

Woolfenden, B. E., Gibbs, H. L., McLaren, C. M. and Sealy, S. G. 2004. Community-level patterns of parasitism: Use of three common hosts by a brood parasitic bird, the brown-headed cowbird. Ecoscience 11:238-248.

Yasukawa, K. and Searcy, W. A. 1995. Red-winged blackbird (Agelaius phoeniceus). In: Poole, A. and Gill, F. (eds.), Birds of North America, no. 184. Acad. Nat. Sci., Philadelphia and Am. Ornithol. Union, Washington.

Zanette, L. and Jenkins, B. 2000. Nesting success and nest predators in forest fragments: a study using real and artificial nests. Auk 117: 445-454. 